\title{
A Low-Complexity DOA Estimation Algorithm for 2D Massive MIMO System
}

\author{
Xiaoyu $\mathrm{Li}^{1, \mathrm{a}^{*}}$, Wei Wu ${ }^{1, \mathrm{~b}}$ and Xiaofei Zhang ${ }^{2, \mathrm{c}}$ \\ ${ }^{1}$ College of Electronic and Information Engineering, Nanjing University of Aeronautics \& \\ Astronautics, Nanjing, China \\ 2 Jiangsu Key Laboratory of Internet of Things and Control Technologies (NanjingUniversity of \\ Aeronautics and Astronautics) \\ axiaoyuLi503@163.com, ’39834290@qq.com, ${ }^{\text {b } z h a n g x i a o f e i @ n u a a . e d u . c n ~}$
}

\begin{abstract}
Keywords: Direction of Arrival (DOA); Massive MIMO; Propagator Method (PM).
Abstract. Massive multiple-input multiple-output (MIMO) system has become a crucial technology meeting the ever-increasing data consumption demand, for it has more channel capacity, higher frequency spectrum efficiency and reliability. The direction of arrival (DOA) estimation on the base station has a marked impact on massive MIMO system capacity and frequency spectrum efficiency. In this paper, we study the DOA estimation method for two-dimensional (2D) massive MIMO system. ESPRIT algorithm has high computational complexity due to the eigenvalue decomposition (EVD) of the signal's covariance matrix. Propagator method (PM) does not require the EVD of the autocorrelation matrix thus needs a relatively small amount of computation. This paper proposes a PM-based 2D DOA estimation algorithm that applies to massive MIMO system. Numerical results demonstrate that the proposed estimation method has lower computational complexity than ESPRIT, and its performance is comparable with ESPRIT in high SNR.
\end{abstract}

\section{Introduction}

Multiple-input multiple-output (MIMO) system utilizes multiple antennas to transmit signals and detect object and has widespread application in radar and wireless communication field [1]. As a promising technology in 5G, massive MIMO has attracted significant attention in academia and industry. Compared with traditional MIMO, massive MIMO brings huge improvements in throughput, link reliability and radiated energy efficiency through the use of a large number of antennas in base station [2-3]. In time division duplex (TDD) cellular system, the base station will rely on DOA estimation to acquire the channel state information (CSI) from uplink sounding signals. For base station (BS) equipped with massive MIMO antenna array, accurate estimation of the direction of arrivals (DOA), including both the elevation and azimuth angles, of source signals is crucial. The achievable rate and the optimal transmission strategy in massive MIMO system depend heavily on the performance of the underlying DOA estimation [4]. There are many existing DOA estimation method for MIMO system such as estimation of signal parameters via rotational invariance techniques (ESPRIT) [5], multiple signal classification (MUSIC) [6], maximum likelihood method [7], Capon algorithm [8], parallel factor (PARAFAC) technology [9], etc. But the DOA estimation on two-dimensional (2D) antenna array is not well explored. An ESPRIT algorithm based on optimal subspace fitting for 2D azimuth and elevation estimation is proposed in [10]. In [11], a 2D iterative adaptive approach based beam-forming algorithm is proposed. Due to the multidimensional searching, these methods have high computational complexity and are not suitable for massive MIMO system. In [12], a low complexity unitary ESPRIT algorithm is proposed to jointly estimate elevation and azimuth angles of the arrived signals in massive MIMO system. In [13], a two-stage low-complexity DOA estimation scheme is proposed, in which two MUSIC algorithms are performed for estimating, respectively, the elevation and azimuth angles of the impinging sources. In [14], an ESPRIT-based method is proposed for 2D localization of incoherently distributed (ID) sources in massive MIMO system. 
The reminder of this paper is structured as follows. Section II describes the system model. Section III introduces the proposed PM-based algorithm and the complexity analysis. Section IV presents numerical simulations to illustrate the performance of our method, and the conclusions are drawn in Section V.

Notations: Lower-case (upper-case) boldface symbols denote vectors (matrices); $(\square)^{T},(\square)^{H},(\square)^{-1}$, $(\square)^{+}$denote the transpose, the conjugate transpose, the inverse, and the pseudoinverse, respectively; $\mathbf{I}_{K}$ is the $K \times K$ identity matrix; $\operatorname{diag}(\square)$ is a diagonal matrix and the values in the brackets are the diagonal elements; angle $(\square)$ is to get the phase.

\section{System Model}

A typical 2D massive MIMO system at the base station is shown in Fig. 1. The planar array is placed in the $X-Z$ plane with $N$ antenna elements in the $X$ dimension and $M$ antenna elements in the $Z$ dimension, respectively. The spacing between adjacent antenna elements is $d$, with $d \leq \lambda / 2$ ( $\lambda$ is the wave length). Assume there are $K$ source signals, say, $s_{i}, 1 \leq i \leq K$ impinging on the BS, each of which has the elevation angle of $\theta_{i}$ and the azimuth angle of $\phi_{i}$. The received signals of the antenna array are

$$
\begin{gathered}
\mathbf{X}=\mathbf{A} \mathbf{S}+\mathbf{N}, \\
\mathbf{A}=\left[\begin{array}{c}
\mathbf{A}_{\mathbf{x}} D_{1}\left(\mathbf{A}_{\mathbf{z}}\right) \\
\mathbf{A}_{\mathbf{x}} D_{2}\left(\mathbf{A}_{\mathbf{z}}\right) \\
\mathbf{M} \\
\mathbf{A}_{\mathbf{x}} D_{M}\left(\mathbf{A}_{\mathbf{z}}\right)
\end{array}\right],
\end{gathered}
$$

where the steering matrix in the $X$ dimension is

the steering matrix in the $Z$ dimension is

$$
\mathbf{A}_{x}=\left[\begin{array}{cccc}
1 & 1 & \mathrm{~L} & 1 \\
e^{j 2 \pi d u_{1} / \lambda} & e^{j 2 \pi d u_{2} / \lambda} & \mathrm{L} & e^{j 2 \pi d u_{K} / \lambda} \\
\mathrm{M} & \mathrm{M} & \mathrm{O} & \mathrm{M} \\
e^{j 2 \pi d(N-1) u_{1} / \lambda} & e^{j 2 \pi d(N-1) u_{2} / \lambda} & \mathrm{L} & e^{j 2 \pi d(N-1) u_{K} / \lambda}
\end{array}\right], u_{i}=\sin \theta_{i} \cos \phi_{i},
$$

$$
\mathbf{A}_{z}=\left[\begin{array}{cccc}
1 & 1 & \mathrm{~L} & 1 \\
e^{j 2 \pi d v_{1} / \lambda} & e^{j 2 \pi d v_{2} / \lambda} & \mathrm{L} & e^{j 2 \pi d v_{K} / \lambda} \\
\mathrm{M} & \mathrm{M} & \mathrm{O} & \mathrm{M} \\
e^{j 2 \pi d(\mathrm{M}-1) \mathrm{v}_{1} / \lambda} & e^{j 2 \pi d(\mathrm{M}-1) \mathrm{v}_{2} / \lambda} & \mathrm{L} & e^{j 2 \pi d(\mathrm{M}-1) \mathrm{v}_{K} / \lambda}
\end{array}\right], v_{i}=\cos \theta_{i},
$$

$D_{i}\left(\mathbf{A}_{z}\right)$ is the diagonal matrix with the diagonal elements being the $i$ th row of the $\mathrm{A}_{z}$

$$
D_{i}\left(\mathbf{A}_{z}\right)=\operatorname{diag}\left\{\left(e^{j(\mathrm{i}-1) v_{1}}, e^{j(\mathrm{i}-1) v_{2}}, \mathrm{~L}, e^{j(\mathrm{i}-1) v_{K}}\right)\right\},
$$

$\mathbf{S}$ is the transmitted signal matrix, $\mathbf{S}=[s(1), s(2), \mathrm{L}, s(L)] \in \square^{K \times L}, L$ is the number of snapshots, $\mathbf{N}$ is the noise matrix $\mathrm{N}=[\boldsymbol{n}(1), \boldsymbol{n}(2), \mathrm{L}, \boldsymbol{n}(L)] \in \square^{K \times L}$.

\section{PM-Based DOA Estimation}

\section{A . Proposed algorithm}

The matrix $\mathbf{A}$ is the concatenation of two sub-matrices $\mathbf{A}_{1} \in \square^{K \times K}$ and $\mathbf{A}_{2} \in \square^{(\mathrm{MN}-K) \times K}$; namely, we have

$$
\mathbf{A}=\left[\begin{array}{l}
\mathbf{A}_{1} \\
\mathbf{A}_{2}
\end{array}\right]
$$

Assume that the array is not spatial fuzzy (namely $\mathbf{A}$ is full column rank) and $\mathbf{A}_{1}$ is nonsingular matrix. Then $\mathbf{A}_{2}$ is the linear transformation of $\mathbf{A}_{1}$

$$
\mathbf{A}_{2}=\mathbf{P}^{H} \mathbf{A}_{1},
$$


where $\mathbf{P} \in \square^{K \times(M N-K)}$ is the propagator operator.

The covariance matrix of the received signals is $\mathbf{R}=\frac{1}{L} \sum_{t=1}^{L} \mathbf{X}(\mathrm{t}) \mathbf{X}^{H}(\mathrm{t})$ and we partition it as

$$
\mathbf{R}=\left[\mathbf{R}_{1}, \mathbf{R}_{2}\right],
$$

where $\mathbf{R}_{1} \in \square^{M N \times K}, \mathbf{R}_{2} \in \square^{M N \times(M N-K)}$.

Since there is noise, the estimator of $\mathbf{P}$ is

$$
\hat{\mathbf{P}}=\left(\mathbf{R}_{1}^{H} \mathbf{R}_{1}\right)^{-1} \mathbf{R}_{1}^{H} \mathbf{R}_{2},
$$

Define

$$
\mathbf{P}_{c}=\left[\begin{array}{c}
\mathbf{I}_{K} \\
\mathbf{P}^{H}
\end{array}\right]
$$

According to (6), (7) and (10), we have

$$
\mathbf{P}_{c} \mathbf{A}_{1}=\left[\begin{array}{l}
\mathbf{A}_{1} \\
\mathbf{A}_{2}
\end{array}\right]=\mathbf{A},
$$

and we divide $\mathbf{P}_{c}$ into two sub-matrices $\mathbf{P}_{c 1} \in \square^{N(\mathrm{M}-1) \times K}$ and $\mathbf{P}_{c 2} \in \square^{N(\mathrm{M}-1) \times K} . \mathbf{P}_{c 1}$ is the first $N(M-1)$ rows of $\mathbf{P}_{c}$, and $\mathbf{P}_{c 2}$ is the last $N(M-1)$ rows of $\mathbf{P}_{c}$. In the same way, we use $\mathbf{A}_{a}$ and $\mathbf{A}_{b}$ to represent the first $N(M-1)$ rows and the last $N(M-1)$ rows of $\mathbf{A}$, respectively.

$$
\left[\begin{array}{l}
\mathbf{P}_{c 1} \\
\mathbf{P}_{c 2}
\end{array}\right] \mathbf{A}_{1}=\left[\begin{array}{c}
\mathbf{A}_{a} \\
\mathbf{A}_{b}
\end{array}\right]=\left[\begin{array}{c}
\mathbf{A}_{a} \\
\mathbf{A}_{a} \mathbf{\Phi}_{z}
\end{array}\right]
$$

where $\boldsymbol{\Phi}_{z}=D_{2}\left(\mathbf{A}_{z}\right)=\operatorname{diag}\left\{\left(e^{j v_{1}}, e^{j v_{2}}, \mathrm{~L}, e^{j v_{K}}\right)\right\}$.

Therefore,

$$
\mathbf{P}_{c 1}^{+} \mathbf{P}_{c 2}=\mathbf{A}_{1} \boldsymbol{\Phi}_{z} \mathbf{A}_{1}^{-1} .
$$

Define

$$
\boldsymbol{\Psi}_{z}=\mathbf{P}_{c 1}^{+} \mathbf{P}_{c 2},
$$

it can be seen that $\boldsymbol{\Psi}_{z}$ and $\boldsymbol{\Phi}_{z}$ have the same eigenvalues, and the eigenvector matrix of $\boldsymbol{\Psi}_{z}$ is $\mathbf{A}_{1}$. We perform the eigen-decomposition of $\boldsymbol{\Psi}_{z}$ and the estimator of $v_{k}$ can be acquired by

$$
\hat{v}_{k}=\operatorname{angle}\left(\lambda_{k}\right) \lambda / 2 \pi d,
$$

where $\lambda_{k}=e^{j 2 \pi d v_{k} / \lambda}$ is the $k$ th eigenvalue of $\boldsymbol{\Psi}_{z}, k=1,2, \mathrm{~L}, K$.

According to (11) we have

Reconstruct $\mathbf{P}_{c}$ to $\mathbf{P}_{c}^{\prime}$

$$
\mathbf{P}_{c}=\left[\begin{array}{c}
\mathbf{A}_{\mathbf{x}} D_{1}\left(\mathbf{A}_{\mathbf{z}}\right) \\
\mathbf{A}_{\mathbf{x}} D_{2}\left(\mathbf{A}_{\mathbf{z}}\right) \\
\mathbf{M} \\
\mathbf{A}_{\mathbf{x}} D_{M}\left(\mathbf{A}_{\mathbf{z}}\right)
\end{array}\right] \mathbf{A}_{1}^{-1} .
$$

$$
\begin{gathered}
\mathbf{P}_{c}^{\prime}=\mathbf{P}_{c} \mathbf{A}_{x 1} \\
\mathbf{P}_{c}^{\prime}=\left[\begin{array}{c}
\mathbf{A}_{z} D_{1}\left(\mathbf{A}_{x}\right) \\
\mathbf{A}_{z} D_{2}\left(\mathbf{A}_{x}\right) \\
\mathbf{M} \\
\mathbf{A}_{z} D_{N}\left(\mathbf{A}_{x}\right)
\end{array}\right] \mathbf{A}_{1}^{-1} .
\end{gathered}
$$

Similarly, we use $\mathbf{P}_{c 1}^{\prime}$ and $\mathbf{P}_{c 2}^{\prime}$ to represent the first $N(M-1)$ rows and the last $N(M-1)$ rows of $\mathbf{P}_{c}^{\prime}$, respectively.

$$
\Psi_{x}=\mathbf{P}_{c 1}^{\prime+} \mathbf{P}_{c 2}^{\prime} .
$$

In theory, $\Psi_{x}$ and $\Psi_{z}$ have the same eigenvector matrix $\mathbf{A}_{1}$,

$$
\boldsymbol{\Psi}_{\mathrm{x}}=\mathbf{A}_{1} \boldsymbol{\Phi}_{\mathbf{x}} \mathbf{A}_{1}^{-1} \text {. }
$$

The estimator of $u_{k}$ can be acquired by

$$
\hat{u}_{k}=\operatorname{angle}\left(\varepsilon_{k}\right) \lambda / 2 \pi d,
$$


where $\varepsilon_{k}=e^{j 2 \pi d u_{k} / \lambda}$ is the $k$ th eigenvalue of $\boldsymbol{\Psi}_{x}, k=1,2, \mathrm{~L}, K$. Finally, the elevation and azimuth angle can be estimated through

$$
\begin{gathered}
\hat{\theta_{k}}=\arccos \left(\hat{v}_{k}\right), \\
\hat{\phi_{k}}=\arccos \left(\hat{u}_{k} / \sqrt{1-\hat{v}_{k}^{2}}\right) .
\end{gathered}
$$

\section{B. Complexity analysis}

Compared to ESPRIT algorithm, the proposed algorithm has lower complexity. $N, M$ denote the number of elements in the dimension of $X$ and $Z$, respectively; $L$ and $K$ denote the number of snapshots and the number of signal sources, respectively. The complexity of the proposed algorithm is $O\left(L M^{2} N^{2}+M N K^{2}+M N(M N-K)+4 K^{3}+K^{2}(M N-K)+2 K^{2}(M-1) N+K^{2} M N+(N-1) M K\right.$, while that of ESPRIT is $O\left(L M^{2} N^{2}+M^{3} N^{3}+2 K^{2}(M-1) N+3 K^{3}+K^{2} M N+(N-1) M K\right)$. The complexity comparison between the two algorithms is shown in Fig. 2 with $K=6, L=100, N=M$. It can be seen that the proposed algorithm has lower computational load than ESPRIT algorithm, and the speed of the computational load increase is much slower than that of ESPRIT with the increase of the number of antenna elements.

\section{The advantages of the proposed algorithm}

- Compared to ESPRIT algorithm, it does not need the eigenvalue decomposition of the signal's covariance matrix, thus has lower computational complexity.

- It can achieve automatic matching of the angle parameters, avoiding the extra computational burden brought about by the matching of the angle parameters.

- In high SNR circumstances, its DOA estimation performance is approaching to that of ESPRIT algorithm.

\section{Performance Evaluation}

In this section, we provide numerical results to illustrate the performance of the proposed approach. The number of simulation trials is 1000 . The spacing between adjacent antenna elements is $d=0.5 \lambda$. The number of antennas in two dimensions is $M=N=16$. The number of snapshots is $L=100$.We consider nine narrowband source signal impinging on the array, with elevation and azimuth angles $\theta=\left\{35^{\circ}, 40^{\circ}, 45^{\circ}, 85^{\circ}, 90^{\circ}, 95^{\circ}, 135^{\circ}, 140^{\circ}, 145^{\circ}\right\} \quad, \phi=\left\{25^{\circ}, 65^{\circ}, 105^{\circ}, 30^{\circ}, 70^{\circ}, 110^{\circ}, 35^{\circ}, 75^{\circ}, 115^{\circ}\right\}$. The DOA estimation performance of the proposed algorithm in different SNRs is shown in Fig. 3 and Fig. 4, respectively. The DOA estimation performance is poor in low SNR, while in high SNR the algorithm can estimate the elevation and azimuth angle accurately.

Given the number of signal sources $K=10$, the root mean square error (RMSE) of elevation and azimuth angle is evaluated for different number of signal sources in Fig. 5. As the figure shows, with the increase of the number of signal sources, the DOA estimation performance is getting worse.

The RMSE of the proposed algorithm in different numbers of snapshots is demonstrated in Fig. 6. The figure shows that the DOA estimation performance is getting better with the increase of the number of snapshots. This is because more accurate signal covariance matrix can be acquired with the increase of the number of snapshots.

Given $K=6$, Fig. 7 compares the RMSE of the proposed algorithm and ESPRIT algorithm. As the figure shows, the proposed method incurs a larger RMSE in low SNR, while in high SNR, the performance of the proposed method is very close to that of ESPRIT algorithm. Although the proposed algorithm has a low complexity at the price of DOA estimation accuracy, its performance is as good as that of ESPRIT algorithm in high SNR. So the proposed algorithm has wider range of application than ESPRIT algorithm. 


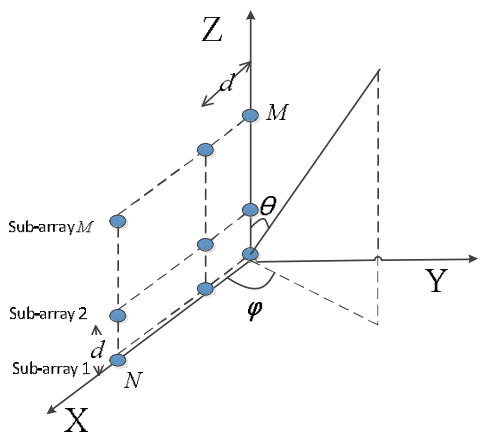

Fig. 1 2D massive MIMO system model

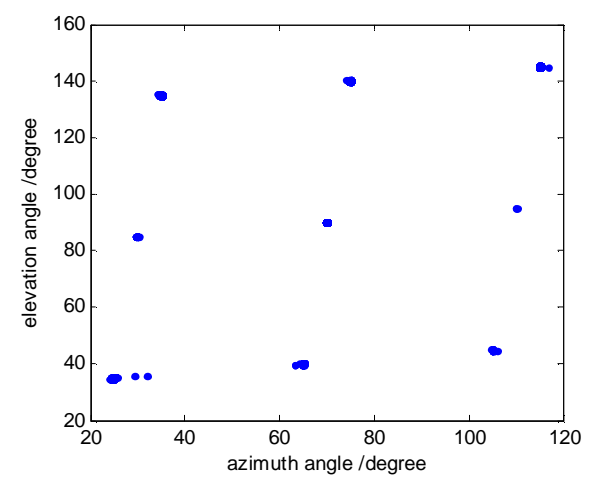

Fig. 3 angle estimation performance $(S N R=10 d B)$

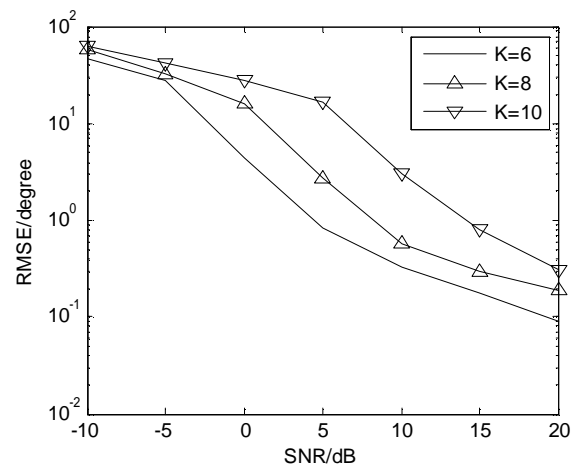

Fig. 5 RMSE performance in different numbers of Fig. 6 RMSE performance in different numbers of signal sources

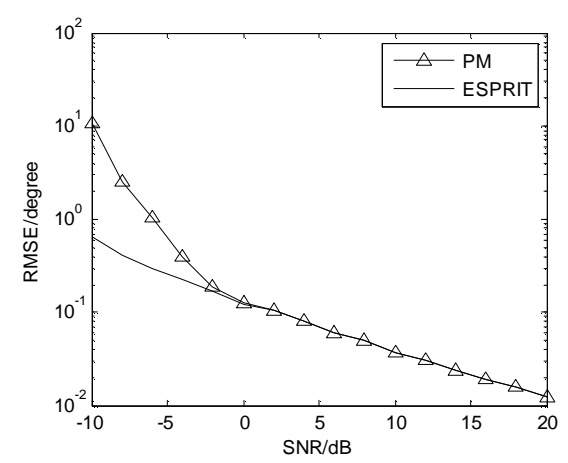

Fig. 7 RMSE performance of the two methods

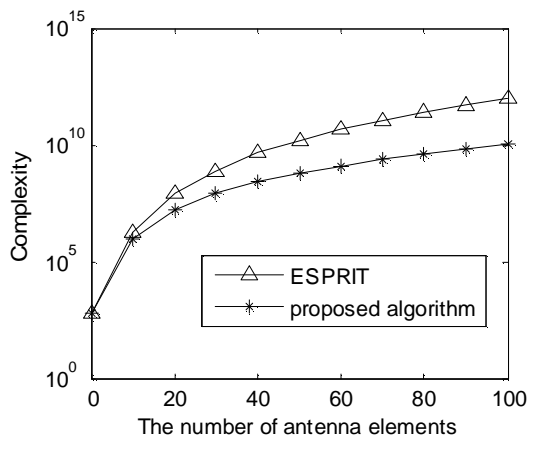

Fig. 2 Complexity comparison between the two algorithms

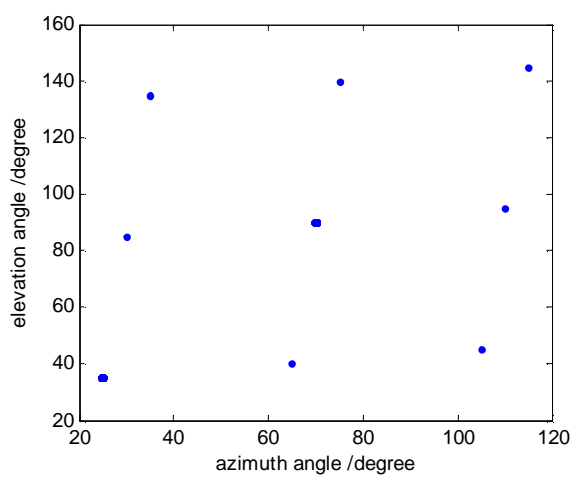

Fig. 4 angle estimation performance $(S N R=20 d B)$

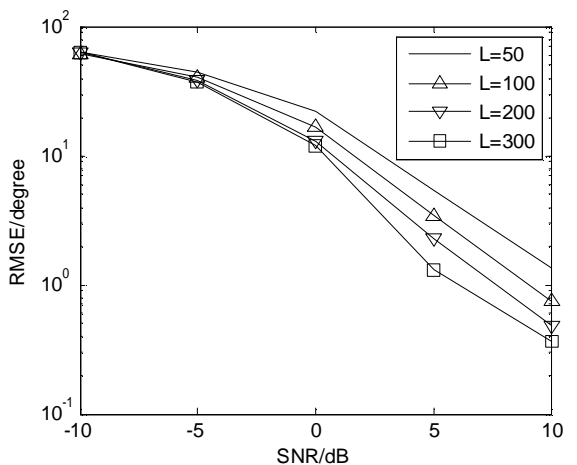
snapshots 


\section{Conclusion}

In this paper we have proposed a low-complexity PM-based DOA estimation scheme for 2D antenna array in the massive MIMO system. The proposed approach does not require to conduct EVD of the signal's covariance matrix, nor does it require peak searching. Simulation results show that it has lower complexity than ESPRIT algorithm, and the performance approaches to ESPRIT algorithm in high SNR.

\section{References}

[1] Fishler E, Haimovich A, Blum R, et al, "MIMO radar: an idea whose time has come," IEEE Radar Conference, 2004:71 - 78 .

[2] Larsson E, Edfors O, Tufvesson F, et al, "Massive MIMO for next generation wireless systems," Communications Magazine IEEE, 2014, 52(2):186 - 195.

[3] Li G Y, Xu Z, Xiong C, et al, "Energy-efficient wireless communications: tutorial, survey, and open issues," Wireless Communications, IEEE, 2011, 18(6): 28-35.

[4] Liu L, Li Y, Zhang J, "DOA estimation and achievable rate analysis for 3D millimeter wave massive MIMO systems," Signal Processing Advances in Wireless Communications (SPAWC), 2014 IEEE 15th International Workshop on. IEEE, 2014: 6-10.

[5] Jinli C, Hong G, Weimin S, "Angle estimation using ESPRIT without pairing in MIMO radar," Electronics Letters, 2008, 44(24):1422 - 1423.

[6] Zhang X, Xu L, Xu L, et al, " Direction of Departure (DOD) and Direction of Arrival (DOA) Estimation in MIMO Radar with Reduced-Dimension MUSIC," Communications Letters IEEE, 2010, 14(12):1161 - 1163 .

[7] Juan Z, Lin-rang Z, Nan L, "Maximum likelihood DOA estimation of MIMO radar," Systems Engineering \& Electronics, 2009, 31(6):1292-1294.

[8] Zhang X, Xu D, “Angle estimation in MIMO radar using reduced-dimension Capon," Electronics Letters, 2010, 46(12):860 - 861 .

[9] Nion D, Sidiropoulos N D, "A PARAFAC-based technique for detection and localization of multiple targets in a MIMO radar system," IEEE International Conference on Acoustics, Speech, and Signal Processing, 2009:2077-2080.

[10] Swindlehurst A L, Kailath T, "Azimuth/elevation direction finding using regular array geometries," Aerospace and Electronic Systems, IEEE Transactions on, 1993, 29(1):145 - 156.

[11] Barcelo M, Vicario J L, Seco-Granados G, "A Reduced Complexity Approach To IAA Beamforming For Efficient DOA Estimation Of Coherent Sources," Eurasip Journal on Advances in Signal Processing, 2011, 37(1):46-51.

[12] Wang A, Liu L, Zhang J, "Low complexity direction of arrival (DOA) estimation for 2D massive MIMO systems," IEEE Globecom Workshops, 2012:703 - 707.

[13] Yang, Kai Yu, J. Y. Wu, and W. H. Li, "A low-complexity direction-of-arrival estimation algorithm for full-dimension massive MIMO systems," Communication Systems (ICCS), 2014 IEEE International Conference on. IEEE, 2014.

[14] Hu, Anzhong, et al, "An ESPRIT-based approach for 2-D localization of incoherently distributed sources in massive MIMO systems." IEEE Journal of Selected Topics in Signal Processing 8.5(2014):996 - 1011. 